\title{
Norois
}

Environnement, aménagement, société

$245 \mid 2017$

Adapter les territoires aux changements climatiques : transition urbanistique et aménagement de l'espace

\section{«Bus à Haut Niveau de Service » (BHNS) : enjeux et perspectives pour une mobilité durable dans la ville de Douala}

"Bus Rapid Transit" (BRT): stakes and prospects for sustainable mobility

in the city of Douala

Claudia Manto Teignegou, André Nso Ngang et Joséphine Mireille

Akoa Etoa

\section{OpenEdition}

Journals

Édition électronique

URL : https://journals.openedition.org/norois/6246

DOI : $10.4000 /$ norois.6246

ISBN : 78-2-7535-7465-6

ISSN : $1760-8546$

Éditeur

Presses universitaires de Rennes

Édition imprimée

Date de publication : 31 décembre 2017

Pagination : 101-111

ISBN : 978-2-7535-7456-4

ISSN : 0029-182X

Référence électronique

Claudia Manto Teignegou, André Nso Ngang et Joséphine Mireille Akoa Etoa, « «Bus à Haut Niveau de Service » (BHNS) : enjeux et perspectives pour une mobilité durable dans la ville de Douala », Norois [En ligne], 245 | 2017, mis en ligne le 31 décembre 2019, consulté le 13 janvier 2022. URL : http:// journals.openedition.org/norois/6246; DOI : https://doi.org/10.4000/norois.6246 


Presses
Universitaires
de Rennes

\title{
«Bus à Haut Niveau de Service » (BHNS) : enjeux et perspectives pour une mobilité durable dans la ville de Douala
}

\author{
"Bus Rapid Transit" (BRT): Stakes and Prospects for Sustainable Mobility \\ in the City of Douala
}

\section{Claudia Manto Teignegou ${ }^{a}$, André Nso $\mathrm{NGang}^{b}$ et Joséphine Mireille Aкоa Etoa ${ }^{\mathrm{c}}$}

\footnotetext{
a Auteur correspondant : Tel : (237) 6757677 08, Économiste des transports, Ministère des Transports/Cameroun

(MINT).(cteignegou@yahoo.fr)

b Socio-économiste, Institut de Recherche Agricole pour le Développement (IRAD) BP 2123 Yaoundé-MessA, Cameroun. (an.nsongang@gmail.com)

${ }^{\mathrm{c}}$ Chercheur : Géographie et aménagement, Institut de Recherche Agricole pour le Développement (IRAD) BP 2123

Yaoundé-Messa, Cameroun. (akoamireille@yahoo.fr)
}

Résumé : Cette étude analyse les conditions de faisabilité du système « Bus à Haut Niveau de Service » (BHNS) dans la ville de Douala, en vue de répondre aux besoins de mobilité des populations et de réduire la pollution atmosphérique liée aux transports dans cette ville. D’après le recensement général de la population de 2005, Douala est la ville la plus peuplé du Cameroun avec une offre de transport en commun majoritairement artisanale. Un peu plus de la moitié des populations de cette ville se déplacent en mototaxis. L'urbanisation rapide et anarchique, l'étalement de la ville, font de la question de mobilité un enjeu aussi bien environnemental que social. Le succès du TransMilenio de Bogota, qui a impulsé le développement du BHNS dans les villes du monde, témoigne du caractère souhaitable de ce système de transport pour la ville de Douala. À Douala, les lignes de transport sont déjà bien définies. Seulement, elles sont exploitées par les minibus et les mototaxis qui relèvent essentiellement du secteur informel (transport artisanal). L'aménagement des sites propres sur ces lignes et la capitalisation de l'expérience des transporteurs artisanaux (exploitants des minibus) à travers leur reconversion dans le BHNS pourrait contribuer au succès de ce système de transport. Il pourra également contribuer au développement durable de la ville dans la mesure où sa meilleure attractivité pourrait réduire le nombre de déplacements réalisés par les mototaxis, les minibus voire les taxis, qui présentent un taux d'émission de polluants plus élevé par passager au kilomètre.

Astract: This study analyzes the feasibility conditions of the Bus Rapid Transit (BRT) in the city of Douala, in order to meet people's mobility needs and reduce air pollution related to transport in this city. According to the general population census of 2005, Douala is the most populous city in Cameroon with a transport supply mainly artisanal. A little more than half of the population of this city moves in motorcycle taxis. The rapid and uncontrolled urbanization, the sprawl of the city, make the mobility an environmental as well as a social issue. The success of the Bogota's TransMilenio which spearheaded the development of BRT in the world's cities, reflects the desirability of this transport system for the city of Douala. In Douala, the transport lines are already well defined. However, they are exploited by minibuses and mototaxis, which mainly belong to the informal sector (artisanal transport). The development of clean sites on these lines and the capitalization of the experience of the artisanal transporters (minibus operators) through their conversion into the BRT could contribute to the success of this transport system. It can also contribute to the sustainable development of the city, since its best attractiveness could reduce the number of trips made by motorcycle taxis, minibuses even taxis, which have a higher pollutant emission rate per passenger per kilometer. 
Mots clés : Changement climatique - mobilité - transport collectif - ville durable - pollution atmosphérique - Cameroun

Keywords: Climate change - mobility - public transport - sustainable city - atmospheric pollution - Cameroon

\section{INTRODUCTION}

Les transports sont un enjeu pour les États africains, aussi bien par les emplois et les activités divers qu'ils génèrent que par leurs répercussions sur l'aménagement des territoires. Ils représentent également un risque pour la planète, avec les menaces relatives au réchauffement climatique dont ils ont une part de responsabilité non négligeable (Dessus, 2009). Principal vecteur d'intégration économique, les services et infrastructures de transport sont le préalable à la facilitation des échanges et à la circulation des biens et des personnes.

Les villes africaines sont caractérisées par un accroissement démographique très rapide accompagné d'un étalement urbain (AFD, 2010). L'absence de politiques d'aménagement des espaces urbains et de développement économique a débouché sur l'extension tentaculaire de ces villes (Kumar et Barrett, 2008).

Au Cameroun, avec l'avènement de la crise des années 1980 et la mise en place des programmes d'ajustement structurels des années 1990, les services de transport public ont été libéralisés dans les grandes villes du pays; avec l'espoir que la concurrence ainsi générée entre les opérateurs conduirait à un système de transport public urbain auto-efficient qui éviterait l'intervention financière des pouvoirs publics (Trans-Africa, 2009). La défaillance du transport public par autobus a plutôt impulsé la prolifération des minibus, des cargos ${ }^{1}$, et des mototaxis qui sont source de congestion et de pollution dans les villes. La ville de Douala en est une parfaite illustration. Avec une population de près de deux millions d'habitants (BUCREP, 2010), elle se présente comme la porte d'entrée du Cameroun et occupe une position stratégique et historiquement dominante dans l'économie du pays. Seulement, circuler à Douala est extrêmement difficile de nos jours, du fait du mauvais état de la voirie, de l'indiscipline des usagers de la route et de l'insuffisance d'une offre

1. Fourgonnette transformé par des petits garagistes locaux en car de transport en commun par des barres de fer, de nature très inconfortable pour le transport des personnes, mais qui sont utilisées à ces fins. de transport public de masse. Cette insuffisance a entrainé l'utilisation accrue des véhicules privés et une prolifération du transport artisanal $^{2}$ (minibus, taxi, cargos, mototaxis...) qui sont à l'origine d'importantes nuisances : la pollution atmosphérique, l'encombrement du trafic, l'insécurité routière, le bruit, etc.

Face à cette situation, le gouvernement camerounais envisage la mise en place d'un système de transport en site propre sur le modèle du Bus à Haut Niveau de Service (BHNS) dans les villes de Douala et de Yaoundé (respectivement capitales économique et politique du pays), afin de répondre d'une part, aux besoins de mobilité des populations et, d'autre part, à la réduction de la pollution atmosphérique issue du trafic routier. Ce projet est encore au stade des études de faisabilité sur les deux lignes pilotes à savoir $33 \mathrm{~km}$ pour la ville de Douala et $18 \mathrm{~km}$ pour la ville de Yaoundé. Au regard de la place importante qu'occupe le transport artisanal dans ces deux villes, et plus particulièrement dans la ville de Douala où les mototaxis assurent $55 \%$ des déplacements motorisés de la ville (CUD, 2009), la question qui se pose ici est : le développement d'un BHNS à Douala est-il souhaitable et faisable? De ce fait, comment mettre en place un système de transport qui intègre au mieux les préoccupations socio-économique et environnementale dans un contexte d'indiscipline caractérisée des usagers de la route et où l'application de la réglementation est presque défaillante?

L'objectif de ce travail est d'envisager tout d'abord les différents enjeux sous-jacents au projet de BHNS dans la ville de Douala, puis d'examiner les pistes de faisabilité, et comment une dimension inscrite dans une perspective de développement durable de la ville pourrait venir s'y insérer.

\footnotetext{
2. Le transport artisanal étant conçu comme l'exploitation à une échelle individuelle de véhicules de transport public dont la propriété est atomisée, c'est-à-dire répartie entre de nombreux propriétaires (Godart, 2008). Selon cet auteur, la notion de transport artisanal insiste sur le caractère éclaté de la propriété des véhicules, mais aussi et surtout sur le haut degré d'autonomie et de responsabilité des chauffeurs et autres personnels de terrain. Généralement informels, ces transports s'opposent aux transports en commun centralisés qui sont d'ordre collectif et institutionnel.
} 
Dans cet article, il s'agit en premier lieu, de faire une analyse de la crise mondiale des transports collectifs dans les pays du Nord et du Sud, des enjeux de la mise en place du BHNS dans une ville voiture ou dans une ville à insuffisance de voirie et à transport artisanal dominant comme Douala. Ensuite, il sera question d'examiner la faisabilité du BHNS dans la ville de Douala et ses possibilités de contribution au développement durable de la ville. Ceci à travers 1) la présentation de la mobilité à prédominance artisanale dans la ville; 2) la nécessité d'une coordination des acteurs institutionnels impliqués dans l'organisation des transports urbains et 3) la présence des lignes de transport «naturelles-spontanées », signes de nécessité et condition d'une mise en place de BHNS, et atout de facilitation de sa faisabilité.

\section{LE BHNS, UNE RÉPONSE À LA CRISE DES TRANSPORTS COLLECTIFS}

\section{Crise des transports collectifs}

Le transport collectif est en crise dans de nombreuses villes, malgré les discours incantatoires en sa faveur, qui viennent d'ailleurs surtout du monde développé qui a donné tant d'importance à l'automobile (Godard, 2008). Dans les pays du Nord, la croissance économique et urbaine des années soixante a entrainé une utilisation accrue de l'automobile. En effet, l'élévation générale du niveau de vie durant les Trente Glorieuses a favorisé la motorisation des ménages, la voiture s’imposant comme un équipement personnel indispensable (Giorgi, 2002). L'évolution des villes, à travers l'augmentation des distances de déplacement entre les périphéries et le centre, a ainsi généralisé l'utilisation des véhicules personnels. D'où le modèle de la « ville voiture » issu de la théorie des «trois âges » de la ville (Zavi, 1976; Dupuy, 1995; Newman et Kenworthy, 1998; Wiel, 1999) qui rend compte de l'impact de la banalisation du transport motorisé individuel sur les organisations urbaines. La construction des routes, des autoroutes et des voies de contournement n'aura pas réussi à limiter l'encombrement des voies. Cette saturation des voies aura un impact négatif sur les transports collectifs qui vont connaître une baisse de la vitesse commerciale ainsi que de leur fréquentation (Bonnafous, 2004). Les villes du Sud, caractérisées par un accroissement démographique très rapide accompagné d'un étalement urbain, ont une évolution qui n'est pas fondamentalement différente de celle des villes du Nord (Halleux, 2015). Pour cet auteur, la théorie des trois âges de la ville, qui se base sur l'évolution des formes urbaines au sein des pays développés, est transférable aux villes du Sud et, en particulier, aux villes des pays les moins avancés où les taux de motorisation demeurent faibles et où l'urbanisation est peu structurée par des réseaux de transports collectifs. Si dans les villes du Nord, la voiture est le principal motif de dé-densification urbaine, dans les villes du Sud, l'étalement urbain est souvent le fait de l'explosion urbaine qui crée un retard évident des transports en commun qui arrivent bien après le bâti (Faye, 2013). Dans les villes africaines, les modes de transport les plus répandus correspondent généralement aux minibus et aux taxis. Ces modes ont souvent remplacé un transport collectif de masse défaillant. Dans ces pays, les entreprises d'autobus peinent à trouver un statut qui leur donne la pérennité pour relever les défis de la croissance urbaine (Godard, 2008). En Amérique latine, $80 \%$ de la population réside dans des métropoles très étendues qui ont peu investi dans les transports publics au profit de l'extension d'un secteur routier rapidement saturé (AFD, 2014). Un certain nombre de solutions y ont toutefois été expérimentées avec succès pour remédier aux problèmes de mobilité dans les villes d'Amérique latine. Parmi ces solutions, celle qui peut s'adapter à plusieurs contextes est le développement des BHNS à Curitiba (Brésil) et à Bogota (Colombie) et qui constitue une source d'inspiration pour les autres villes du monde en quête des solutions pour la relance ou le développement des transports en commun de par son coût relativement limité pour un niveau de service assez proche du niveau de service assuré par un métro.

\section{Le BHNS comme outil de relance et de développement des transports en commun}

Le BHNS est un système de transport né à Curitiba (Brésil) en 1974 (Castonguay, 2012) qui a commencé à se rependre dans le monde avec le succès fulgurant du TransMillenio ${ }^{3}$ de Bogota

\footnotetext{
3. Castonguay défini le transMilenio comme « un système de transport collectif à voies exclusives alimenté par des autobus à articulation simple ou double inspiré du modèle inauguré à Curitiba au Brésil en 1974 ».
} 
(Colombie) dans les années 2000 (Godard, 2008). Le tableau 1 en fait illustration en présentant l'évolution de sa mise en place dans les villes du monde. Son apparition est récente en Afrique; trois villes en sont déjà équipées et 12 villes l'ont encore sous forme de projet.

Le BHNS est « un mode de transport rapide, flexible et performant qui combine une variété d'éléments physiques et opérationnels en un système intégré de façon permanente avec une image de qualité et une identité unique » (Arnold et al., 1995). C'est un système complexe qui permet de répondre aux besoins de mobilité. Pour des capacités et des vitesses de transport du même ordre, il est beaucoup moins cher, en investissement initial qu'un métro (Giraud, 2008). Sa particularité réside sur le fait qu'il utilise les voies réservées de bus qui permettent d'augmenter la vitesse commerciale de ceux-ci.

\section{Quelques exemples de BHNS}

\section{Le TransMilenio de Bogota}

L'ancien système de transport de Bogota, Bolivie, était composé de lignes de bus privées inefficaces et de réseaux informels de minibus, entraînant une pollution atmosphérique locale et des émissions de $\mathrm{CO}_{2}$ élevées. La mise en place du TransMilenio dans cette ville a contribué à réduire les bruits de 3-10 décibels et à faire baisser le nombre d'accidents de la route d'environ $50 \%$ (Carvajal et al., 2015). Selon un rapport de la Banque mondiale (2002), le TransMilenio a entraîné une réduction de 40 \% de certains polluants atmosphériques à Bogota entre son lancement en décembre 2000 et mai 2001. Il a été enregistré comme premier projet de transport du Mécanisme de Développement Propre (MDP) en 2006 (Grütter, 2006).

\section{Le Dar es Salaam Rapid Transit (DART)}

À Dar es Salaam, Tanzanie, le transport public est assuré par des bus privés dits « daladala » avec une forte congestion du trafic. Le Dar es Salaam Rapid Transit (DART) est actuellement mis en place dans le but et l'ambition de fournir un meilleur service de transport en commun, plus moderne et peu polluant pour les habitants de la ville. Le projet est divisé en 6 phases comprenant chacune la construction d'un « corridor» stratégique (BAD, 2015). La première phase du projet, d'une vingtaine de kilomètres de longueur, avec des voies réservées aux autobus, a déjà été réalisée avec un total de 29 stations, et un service de bus a été déployé au début de l'année 2016. L'ensemble du système est exploité par Salaam Rapid Transit de Usafiri Dar (UDA-RT), sous la surveillance de la surface et l'autorité de réglementation du transport maritime (Sumatra). Ce système a un double objectif. Le premier est la réduction de la congestion urbaine et donc de la pollution. Le second est celui de faire de Dar es Salaam un modèle en Afrique de l'Est et de donner une image très positive de la ville.

\section{Le Bus à Haut Niveau de Service (BHNS) de Lagos (figure 1 - planche IX)}

Avec près de 17 millions d'habitants, Lagos est la capitale commerciale du Nigéria (Mobereola, 2009). En l'absence d'un réseau de transport en commun structuré, le transport en commun est essentiellement assuré par des exploitants individuels avec des autobus de très mauvaise qualité. En vue d'améliorer les transports publics, les autorités nigérianes ont développé un BHNS « léger » à Lagos. Ce BHNS est dit « léger » car, moins sophistiqué que le TransMilenio de Bogota, il est adapté

\begin{tabular}{|c|c|c|c|c|c|c|}
\hline Continents & $1970-1979$ & $1980-1989$ & $1990-1999$ & $2000-2009$ & $2010-2015$ & Total \\
\hline Asie & 0 & 0 & 2 & 17 & 20 & 39 \\
\hline Afrique & 0 & 0 & 0 & 2 & 1 & 3 \\
\hline Amérique du Nord & 1 & 1 & 3 & 22 & 12 & 39 \\
\hline Amérique du Sud & 5 & 7 & 4 & 12 & 22 & 50 \\
\hline Europe & 3 & 1 & 10 & 32 & 10 & 56 \\
\hline Océanie & 0 & 1 & 0 & 5 & 0 & 6 \\
\hline
\end{tabular}

Tableau 1 : Évolution des BHNS dans les villes du monde de 1970 en 2015 sans distinction entre BRT et BHNS (d'après brtdata.org) Evolution of the BRT in the cities of the world from 1970 to 2015 
au contexte africain avec des tarifs abordables, tout en préservant le plus possible les caractéristiques fondamentales du concept de BHNS. Avec un transport de 200000 passagers (Mobereola, 2009) au quotidien, la vitesse moyenne des véhicules est passée de moins de $15 \mathrm{~km} /$ heure à $25 \mathrm{~km} /$ heure.

Le développement d'un système de transport urbain de masse intégré requiert une attention coordonnée à la planification, à la construction et à l'entretien des infrastructures ainsi qu'à l'organisation des services de transport (Kumar et Barrett, 2008). Cependant, l'implication de plusieurs administrations au niveau de la planification, de la réglemen- tation et de la gestion de l'activité en est un frein comme tel est souvent le cas dans les pays africains.

\section{L'Autorité Organisatrice des Transport (AOT) comme outil d'intégration institutionnelle}

Une intégration institutionnelle par la création d'une AOT permet 1) de réduire le risque de concurrence entre modes de transport, 2) la coordination des interventions des différents acteurs à travers la définition de politiques de mobilité et 3) la programmation des investissements (Allaire et al., 2015) (Voir encadré).

\section{Le BRT est-il faisable dans la ville de Douala?}

Pour Allaire et al. (2015), une AOT est une structure métropolitaine qui intègre un grand nombre de compétences associées à la mobilité urbaine dont l'efficacité dépend de plusieurs paramètres :

Une base légale. Les fondements juridiques de l'AOT peuvent être différents. L'institution peut être créée par la loi ou par un accord entre collectivités locales. Elle peut avoir un statut public mais aussi dépendre du droit privé. Elle peut également être le service d'une municipalité voire d'une administration d'État. Étant donné les délais possibles de création, une structure plus informelle peut précéder la création de l'institution.

Le périmètre des autorités responsables du transport. Souvent le territoire concerné par les déplacements des personnes et des marchandises couvre un périmètre impliquant plusieurs municipalités et il est important de déterminer quel est le périmètre d'intervention pertinent, à partir d'enquêtes origine-destination des personnes mais également des flux liés à l'activité économique. Ce périmètre pourra d'ailleurs évoluer dans le temps.

La répartition des compétences. Les pouvoirs donnés aux autorités en charge des transports peuvent se limiter à l'organisation des transports collectifs (tous modes confondus) mais également associer la gestion de la circulation, du stationnement, des modes actifs, etc. Dans une AOT très intégrée, l'institution peut également avoir un rôle dans la planification urbaine et l'urbanisme. Les conditions politiques ne sont pas toujours réunies pour doter une AOT de l'ensemble de ces fonctions et une progressivité est souvent nécessaire. L'intégration des compétences peut donc se faire par étapes avant de trouver l'organisation qui convient le mieux à l'histoire et à la culture institutionnelle de chaque pays, de chaque ville.

Les moyens financiers. L'AOT devra disposer de ressources financières stables et garanties afin de mettre en œuvre la politique de déplacements qu’elle élabore. L'autorité peut soit bénéficier de ressources provenant de l'État ou d'autres collectivités locales, soit disposer de sa propre fiscalité. Afin de porter une politique sur le long terme, il est essentiel que l'AOT dispose d'une bonne visibilité sur ses ressources.

Le besoin de coordination. En l'absence d'une AOT et pour qu'il y ait cohérence dans l'organisation générale du système de transport, il est important qu'existent des structures de coordination entre les différentes institutions intervenant dans le domaine du transport urbain. Ainsi, les provinces, régions ou états fédérés selon les organisations administratives et politiques, sont souvent investies d'un rôle d'aménagement du territoire et peuvent intervenir dans l'organisation et le financement du transport urbain. 
Si le BHNS est déjà mis en exploitation dans quelques villes africaines (Lagos, Dar Es Salam, etc.) il est encore au stade de projet dans plusieurs autres. Le gouvernement camerounais, quant à lui, envisage la mise en place du BHNS dans les villes de Douala et Yaoundé, en prélude à la Coupe d'Afrique des Nations (CAN) qui aura lieu en 2019. Nous allons maintenant nous interroger sur la faisabilité de ce projet dans la ville de Douala, la plus grande ville du Pays.

\section{FAisabilité de LA Mise EN PLACE DU BHNS DANS LA VILLE DE Douala}

De nombreuses pensées militent en faveur du BHNS comme nouvel outil de développement des transports en Afrique. Pour Giraud (2008), le BHNS est particulièrement bien adapté à beaucoup de villes du Sud car, " pour des capacités et des vitesses de transport du même ordre, il est beaucoup moins cher en investissement initial, qu'un métro ». Pour Godard (2008), c'est un système qui permet de restructurer l'ensemble du secteur des transports mais dont la mise en place dans les villes africaines est confrontée à la faiblesse des institutions et aux modalités d'association des transporteurs artisanaux. Il est question ici d'examiner la faisabilité de la mise en place du BHNS dans la ville de Douala face à ces deux contraintes.

\section{Douala : une offre de transport majoritairement artisanale}

Dans la ville de Douala, l'offre de transport en commun est constituée de bus, de minibus, de taxis et de mototaxis. D’après la figure 2 , issue des résultats des enquêtes d'origine-destination réalisées par la communauté urbaine de Douala (CUD) dans le cadre du Plan de Déplacement Urbain (PDU), la société de bus SOCATUR assure à peine $0,5 \%$ des déplacements de la ville. Avec une part de $55 \%$, les mototaxis génèrent une grande partie des déplacements en ville, suivis des voitures particulières et des taxis avec des parts égales de $19 \%$ (CUD, 2009).

La prolifération des mototaxis dans la ville de Douala a été impulsée par les « opérations villes mortes » des années quatre-vingt-dix, les multiples mouvements d'humeurs des chauffeurs de taxi, le chômage, la dégradation de la voirie et l'insuffisance du transport urbain de masse. Peu à peu, la moto s'est imposée comme mode de transport dans la ville (Kaffo et al., 2006). L'attrait des populations de Douala pour le mototaxi pourrait s'expliquer par sa capacité à se faufiler entre les voitures sur les voies congestionnées de la ville, ce qui rend la moto plus rapide par rapport aux autres modes de transport malgré le risque élevé des accidents. En effet, la moto a l'avantage d'occuper moins de place dans un système urbain soit pour se garer, soit dans le trafic par rapport à une automobile (Lefèvre, 2009). Ceci fait de la moto un choix

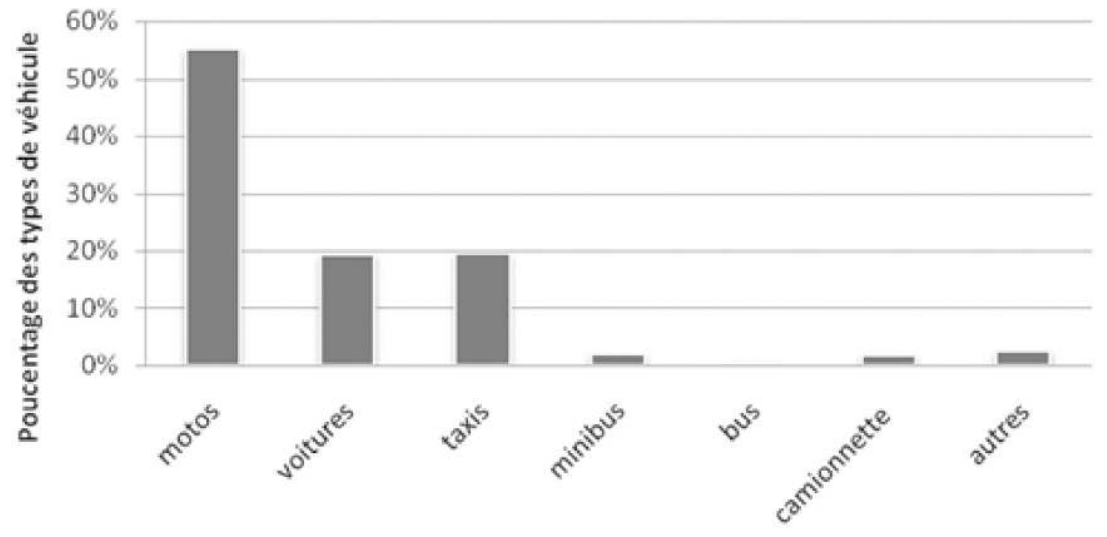

Types de véhicule
Figure 2 : Distribution modale du trafic journalier motorisé à Douala (données CUD 2009) torized traffic in Douala 
logique lorsque l'espace de la rue est limité comme tel est le cas dans la ville de Douala. Contrairement aux minibus qui desservent des lignes de transport bien déterminées, la moto a l'avantage de circuler partout dans la ville, et cela malgré les efforts de la Communauté Urbaine qui visent à interdire son accès au centre-ville. Les utilisateurs de taxis quant à eux estiment que le taxi est un moyen de transport plus sûr et confortable que les motos et les minibus (Berger, 2009). Les autobus sont le mode de transport urbain le moins convoité par les usagers de la route. En effet, depuis la faillite de la Société Camerounaise des Transports Urbains (SOTUC) en 1994, les sociétés de transport urbain de masse ont du mal à se maintenir dans les villes camerounaises. Le vide laissé par le transport urbain de masse a été comblé par le transport informel. Le mauvais état des voiries, les coûts élevés de maintenance, la concurrence du transport informel et l'encombrement du trafic constituent une entrave pour le développement du transport de masse. De plus, dans les embouteillages, les transports publics sont plus lents que les véhicules privés, de sorte que seules les personnes qui peuvent acheter une voiture ou une moto peuvent augmenter leur vitesse de déplacement (Lefèvre, 2009). Le BHNS pourrait-il faire face à ces concurrences?

Dans la ville de Douala, les lignes de transport sont spontanément définies bien qu'étant en majorité exploitées par le transport artisanal. L'aménagement des voies réservées de bus sur ces lignes pourrait augmenter leur vitesse commerciale et rendre le transport en commun beaucoup plus attrayant qu'il ne l'est actuellement. Toutefois, pour une intégration physique et une coordination de l'offre de transport, le BHNS pourrait capitaliser l'expérience de terrain des transporteurs artisanaux. De ce fait, certains transporteurs informels pourront être reconvertis vers la société de bus, et d'autres pourront être chargés d'un service de proximité, intégré à la circulation, qui dessert les zones plus périphériques de la ville. Cependant, toutes ces actions ne pourront être réalisées avec succès que si la réglementation est strictement appliquée et le cadre institutionnel clairement défini.

\section{Multiplicité des acteurs institutionnels du transport urbain et absence de coordination entre les différentes interventions}

Au Cameroun, vers les années 1980, contraint par la conjoncture économique, l'État a voulu par les politiques d'ajustement structurel laisser la gestion du transport aux particuliers, en vue de protéger le secteur et d'assurer du travail à tous. Le secteur des transports est devenu un refuge pour les chercheurs d'emplois et le secteur informel. La souplesse des lois et la tolérance administrative vis-à-vis de l'application des textes réglementaires ont favorisé l'entrée dans le secteur des transports de personnes n'ayant aucune connaissance professionnelle en la matière. Il en a résulté une prolifération du transport informel dans les villes du pays, plus particulièrement dans la ville de Douala qui compte $20 \%$ de la population urbaine du Cameroun (CUD, 2009).

Au Cameroun, plusieurs administrations sont chargées de l'organisation, de la réglementation et de la planification des transports urbains. Le ministère des Transports joue le rôle de régulateur à travers la définition et la mise en œuvre des politiques de transport. Les Communautés Urbaines ont pour responsabilité la protection et l'entretien du patrimoine routier et l'organisation des trafics routiers dans les centres urbains. Le ministère de l'Habitat et du Développement urbain, quant à lui, est chargé entre autres de l'élaboration et de la mise en œuvre des stratégies de gestion des infrastructures urbaines, de l'élaboration et de la mise en œuvre des stratégies d'amélioration de la circulation dans les grands centres urbains. Si le développement d'un système de transport urbain de masse requiert une attention coordonnée à la planification, à la construction et à l'entretien des infrastructures ainsi qu'à l'organisation des services de transport (Kumar et Barrett, 2008), l'implication de plusieurs administrations au niveau de la planification, de la réglementation et de la gestion de l'activité reste un frein pour ce développement. Malgré la définition des responsabilités des différentes administrations par les textes comme nous l'avons vu ci-dessus, on assiste le plus souvent à une confusion des rôles qui se traduit par ce que Kumar et Barrett (2008) ont appelé une mauvaise responsabilisation et une dilution des engagements vis-à-vis de la mise en œuvre 
des stratégies de transport. La confusion des rôles rend la réglementation inefficace. D'où la nécessité d'une intégration institutionnelle par la création d'une instance de coordination qui réunit tous les acteurs locaux, régionaux et nationaux impliqués dans le transport et les déplacements urbains de la ville de Douala.

\section{Contribution du BHNS au développement durable de la ville}

L'aménagement des sites propres permet de réduire le nombre total de véhicules nécessaires pour assurer un niveau de service donné. Il contraint à cet effet la circulation des autres modes de transport par la réduction de l'espace des voies de circulation disponibles et donc encourage l'utilisation des transports en commun. Il a le potentiel de structurer l'ensemble de l'offre de transport et de planification urbaine comme l'illustre la figure 3.

Dans le cas de Douala, la meilleure attractivité du BHNS, due à l'augmentation de la qualité de service (gains de temps, fiabilité des temps de parcours et confort), pourrait réduire le nombre de déplacements réalisés par les mototaxis, les minibus voire les taxis, qui présentent un taux d'émission de polluants plus élevé par passager au km.

Deux corridors est-ouest ont été identifiés pour l'aménagement des sites propres dans la ville. Ces deux corridors ont été complétés par la suite par un corridor de raccordement nord-sud. Le choix des corridors a été fait en prenant en compte les éléments de facilité d'implantation et d'opération, mais aussi la demande en transport. Ce choix a été orienté par l'analyse des études antérieures réalisées dans la ville (Schéma directeur d'urbanisme de Douala à l'horizon 2025) et par une décision du Gouvernement camerounais visant à desservir le nouveau stade Japoma pendant la CAN de 2019. La figure 4 (planche IX) illustre les trois tracés choisis à savoir :

- Les deux corridors est-ouest : l'un qui va connecter la zone du port de Douala avec les bassins de demande entre Douala, Bepanda, Cité des Palmiers et les universités (en jaune), et un autre au Sud du premier corridor, qui va connecter l'aéroport international au futur stade Japoma, aux proximités de la rivière Dibamba, au sud-est de Douala (en bleu);

- Le corridor de raccordement Nord-Sud (en orange).

\section{Conclusion}

Cet article analyse les enjeux, la faisabilité du BHNS et sa contribution au développement durable de la ville de Douala. L'augmentation de l'utilisation des voitures personnelles, l'insuffisance de l'offre de transport en commun et de la voirie ont été à l'origine de la crise des transports collectifs qui pose un problème de mobilité dans la plupart des villes du Sud. Le succès du TransMillenio de Bogota a été une source d'inspiration pour la relance

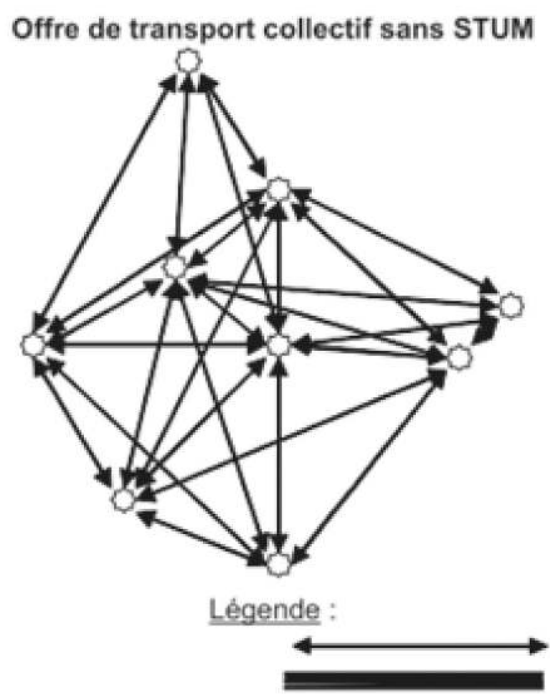

: Flux de déplacements non assurés par un STUM : Flux de déplacements assurés par un STUM

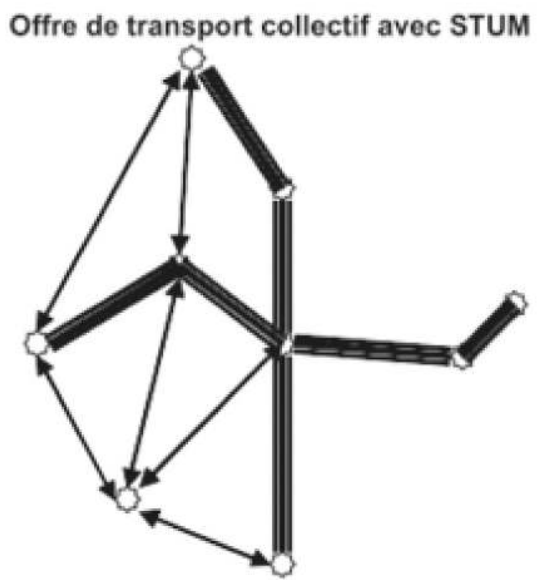

108
Figure 3 : Système de Transport Urbain de Masse (STUM) comme outil de restructuration de l'offre de transport collectif urbain (d'après Plan de Déplacements Urbains-ville de Yaoundé/Rapport mission2) Mass Urban Transport System (MUTS) as a restructuring tool for urban public transport supply 
et le développement du transport collectif des villes. En Europe, les sites propres sont de plus en plus aménagés sous le nom de BHNS. En Afrique, on a notamment l'exemple du BHNS light de Lagos et de celui de Dar Es Salam. En Union indienne, il y a le «Janmarg » qui est le BHNS d'Ahmedabad. Le succès du BHNS dans ces villes milite en faveur du caractère souhaitable de la mise en place de ce système de transport dans la ville de Douala au vu de son étalement spatial, de l'engorgement de son trafic, source de pollution atmosphérique, étant entendu qu'elle présente déjà des avantages naturels quant à l'existence des lignes de transport spontanément définies.

Cependant, s'il est vrai que le BHNS est une solution louable pour la mobilité dans les villes, sa mise en place à Douala, par contre, est conditionnée par : 1) une intégration institutionnelle à travers la création d'une entité en charge de la coordination des différentes actions des administrations impliquées dans l'organisation des transports dans cette ville;2) une coordination de l'offre de transport par la capitalisation de l'expérience de terrain des transporteurs artisanaux à travers la reconversion de certains vers la société de bus et le report des autres vers un service de proximité intégré à la circulation desservant les zones périphériques de la ville. S'agissant de la contribution du BHNS au développement durable de la ville, son attractivité pourrait réduire le nombre de déplacements réalisés par les mototaxis, les minibus, voire les taxis qui présentent un taux d'émission de polluants plus élevé par passager au kilomètre.

\section{Bibliographie}

AFD, 2014. Mobilité dans les pays d'Amérique latine, les rencontres du développement, $25 \mathrm{p}$.

AFD, 2010. Les interactions entre formes urbaines et transport dans la perspective d'un développement urbain soutenable, table ronde, Paris, $38 \mathrm{p}$.

Allaire J., Martinie M., Tomasoni L., Hoyez M., Souirgi R., 2015. La mobilité urbaine émettrice des solutions contre le dérèglement climatique, AFD/CODATU, 76 p.

Arnold B., Van Deursen A., Res M., 1995. An algebraic specification of a language for describing financial products, Eindhoven University of Technology, Departement of Mathematics and Computing Science, ISSN 0926-4515, 17 p.
BAD, 2015. Dar es Salaam Bus Rapid Transit, Environnemental and Social Impact Assessment (ESIA) Infrastructure Project, Tanzania, no P-TZ-DB0-021, OITC.2, 31 p.

Bonnafous A., 2004. Le choix entre voiture et transport collectif in Institut des Villes (éd.) Ville et économie, Paris, La Documentation française, coll. «Ville et société », ISBN 2-11-005569-3, p. 185-206.

Brun G., 2002. La mobilité urbaine: questions récurrentes et réponses de la recherche, Economie E Humanisme, n 359 , $6 \mathrm{p}$.

BUCREP, 2010. Présentation des résultats définitifs $d u 3^{e}$ Recensement Général de la Population et de l'Habitat, Cameroun, $10 \mathrm{p}$.

Carvajal G., Castro H., Garcia M., Prieto F., 2015. Perspectiva desde el enfoque humanista al Plan de desarrollo de Bogotá: Economista en formación, Universidad Santo Tomás, Bogotá, Colombia, DC 2012-2016, 15 p.

Castonguay J.F.M., 2012. Le TransMilenio de Bogota: un outil de lutte contre la pauvreté? Maîtrise en Urbanisme, Université de Montréal, 94 p.

CUD, 2009. Projet d'infrastructure de Douala : élaboration d'un plan de transport et de déplacements urbains de la ville de Douala, Rapport final, 267 p. (non publié).

Dupuy G., 1995. Les territoires de l'automobile, in l'information géographique, Persée, 61 (1), p. 41.

Dessus B., 2009. Les transports face aux défis de l'énergie et du climat, AFD, Document de travail, 86, 110 p.

FAYE D., 2013. Urbanisation et dynamique des transports « informels » et des mobilités dans les villes secondaires sénégalaises: les cas de Touba, Thiès et Saint Louis. Thèse de doctorat en géographie, Bordeaux 3, Université Michel de Montaigne, $270 \mathrm{p}$.

Giorgi F., 2002. Prise en compte des transports en commun de surface dans la modélisation macroscopique de l'écoulement $d u$ trafic. Thèse de Doctorat en génie civil, Institut National des Sciences Appliquées de Lyon, 334 p.

Giraud P.-N., 2008. La croissance urbaine soutenable des villes du Sud. Quelques remarques, Annales des Mines - Réalités industrielles, p. 32-36. DOI 10.3917/rindu.081.0032.

Godard X., 2008. Variété des systèmes de mobilité urbaine face aux enjeux de développement et d'environnement, Colloque international Environnement et transports dans des contextes différents, Algérie, Ghardaïa, p. 144-159.

Grütter J., 2006. BRT Bogotá, Colombia: TransMilenio phase II-IV, Grütter Consulting on behalf of Corporación Andina de Fomento (CAF) and TransMilenio SA, 82 p.

Halleux J-M., 2015. Les territoires périurbains et leur développement dans le monde : un monde en voie d'urbanisation et de périurbanisation, Territoires périurbains, Développement, enjeux et perspectives dans les pays du Sud, p. 43-61.

Kaffo C., Kamdem P., Tatsabong B., 2006. L'intégration des "Motos-taxis » dans le transport public au Cameroun ou l'informel à la remorque de l'État: une solution d'avenir au problème de mobilité et de l'emploi urbain en Afrique subsaharienne, $17 \mathrm{p}$. 
Kumar A., Barrett F., 2008. Coincés dans les embouteillages: Le transport urbain en Afrique. Diagnostics des infrastructures nationales en Afrique, Banque Mondiale et SSAPT, 12 p.

Lefèvre B., 2009. Urban Transport Energy Consumption: Determinants and Strategies for its Reduction. An analysis of the literature. Surveys and Perspectives Integrating Environment and Society, Vertigo, Cities and Climate Change, 2 (3), 18 p.

Marcopolo et MINT, 2016: projet de mise en place des corridors pilotes de BRT dans la ville de Douala (non publié).
Mobereola D., 2009. Premier bus à haut niveau de service en Afrique le «BRT-Lite » de Lagos, document d'analyse SSATP $\mathrm{n}^{\circ}$ 9, série transport urbain, $55 \mathrm{p}$.

Newman P.W.G., Kenworthy J.R., 1998. Sustainability and cities. Overcoming automobile dependence, Island Press, Washington D. C., Covelo, California, 447 p.

WIEL M., 1999. La transition urbaine : Villes bonnes à vivre, villes invivables, MAUSS, 14, p. 175-184.

Zahavi Y., 1976. Travel characteristic cities of developing and developed countries, AGRIS, FAO. 